P9 (continued)

Background: The only known treatment for celiac disease (CeD), a common autoimmune disease, is a glutenfree (GF) diet - where strict adherence has been associated with improved quality of life (QOL).

Objective: To determine whether diet quality or ultraprocessed food (UPF) consumption was associated with QOL in National Health and Nutrition Examination Survey (NHANES) and in a sample with CeD.

Study Design, Settings, Participants: A cross-sectional analysis of a sample of 50 adults $(84 \% \mathrm{~F})$ and 30 teens $(80 \% \mathrm{~F})$ with CeD from a regional CeD-Center in a Northeastern American city was compared with three 2year cycles (2009-14) of NHANES, 15,853 adults (51\% F) and 2,304 teens (52\% F), including individuals with CeD.

Measurable Outcome/Analysis: Diet quality using Alternate Mediterranean Diet (AMED) and level of UPF (\% energy) using the NOVA classification system, were assessed using multiple 24-h diet recalls. All foods, even GF, were categorized. QOL was measured using 5 components of the CeDspecific QOL survey (CDQOL) - adult sample with CeD, the CeD-specific Pediatric - QOL (CDPQOL) - teen sample with $\mathrm{CeD}$, and a general health-related QOL (HRQOL) survey NHANES. Associations between diet quality and QOL were examined using ordinal logistic regression for the NHANES data, and ANCOVA for the smaller sample $(P<0.05)$.

Results: In NHANES, being in the highest versus lowest of 3 AMED categories, or lowest versus highest quintiles of UPF consumption, were $\sim 2 \mathrm{x}$ more likely to have higher QOL, explaining 8-10\% of variance. Among the smaller teens sample with $\mathrm{CeD}$, those in the highest versus lowest AMED tertile were more likely to have higher QOL, but no association was found in adults. Additionally, for all in the smaller sample with $\mathrm{CeD}$, those in the lowest versus highest tertile of UPF were more likely to have higher QOL (some components). All $P<0.05$.

Conclusion: Higher adherence to AMED and lower UPF consumption were associated with higher QOL in NHANES and less robustly in a smaller sample with CeD.

Funding: NIH Provost Investment Fund, Teachers College, Columbia University.

\section{P10 Child Well-Being in the Context of Food Insecurity: A Nutritional Assessment of the Secret Meals for Hungry Children Program}

BailiGall, BS, bjgall@crimson.ua.edu, University of Alabama, 350 Marrs Spring Rd, Tuscaloosa, AL, 35401; Emily Locke, BA, University of Alabama; Holly Horan, PhD, University of Alabama

Background: In Tuscaloosa, Alabama, $19.8 \%$ of children currently experience food insecurity. To reduce the negative health outcomes associated with childhood food insecurity, West Alabama Food Bank (WAFB) distributes food to vulnerable children in elementary schools through their Secret Meals for Hungry Children program (SMP). This program ensures nutritional continuity over the weekend. There are no nutritional guidelines for the SMP meals; however, WAFB aims to provide food items based on the Dietary Guidelines for Americans (DGA).

Objective: The objective of this pilot study was to determine the degree to which the nutritional content of the food distributed in the SMP by WAFB meets the nutritional guidelines as outlined by the 2020-2025 DGA.

Study Design, Setting, Participants: This study implemented an exploratory research design via qualitative and quantitative methods, including photos and nutrition label content analysis. Two qualitative interviews were conducted with WAFB leadership to contextualize the SMP.

Measurable Outcome/Analysis: Carbohydrates, protein and sodium content for the items in the SMP bag were compared to the DGA for children 4-8 years of age to determine if the contents sufficiently supplemented daily recommendations. Semi-structured interviews were analyzed in NVivo $12^{\circledR}$ and themes were identified.

Results: All 3 measures of nutrition (carbohydrates, protein, and sodium) comprised $89 \%, 78 \%$ and $68 \%$ of the recommended guidelines for a child 4-8 years of age, respectively. Two key themes arose from the interviews: SMP organization and SMP impact on children's level of food security and overall wellbeing.

Conclusion: The results indicate that the SMP, according to the DGA, fills the hunger gap for children experiencing food insecurity. SMP provisions were reported to improve overall behavior and functioning at school. These demonstrate the significance of supplemental meals with respect to enhancing behavioral and academic performance for children from vulnerable communities. The implementation of such programs may decrease inequities in learning and overall well-being.

Funding: None.

\section{P11 Consumer Attention to Facts Up Front Labels on Similar Products with Different Serving Sizes}

Gina McKeon, MS, Rutgers University; William Hallman, PhD, gina.pope@rutgers.edu, Rutgers University, 65 Dudley Rd, New Brunswick, NJ, 08901

Background: Facts Up Front (FUF) nutrition labels are used by food manufacturers to highlight selected nutrition information on the front of the package. Differences in serving sizes between similar products may go unnoticed by consumers, leading to misinterpretations of the nutritional information displayed in the FUF.

Objective: To examine consumer attention to FUF labels on 2 similar cereal products with differing serving sizes.

Study Design, Setting, Participants: Utilizing a nationally representative sample, 1,022 adults participated in an online survey.

Measurable Outcome/Analysis: Participants were shown images of 2 cereals with FUF labels that differed in serving size and nutrient amounts. Attention to the FUF 


\section{P11 (continued)}

and serving sizes was assessed, and participants were asked comparative questions about the nutrient content of the cereals. Descriptive statistics and multinomial logistic regressions were performed.

Results: While $64.5 \%$ of participants reported looking at the FUF, only $21.3 \%$ were correct when asked which of the 2 cereals had the bigger serving size. The majority of participants incorrectly indicated that there were no differences between the cereals with respect to the nutrients presented, though these inaccuracies were less likely among participants who looked at the FUF. What seemed to go unnoticed by most participants were the marked nutritional differences between the products if the servings are equalized to 1 cup. In fact, when the serving sizes are equalized, the cereal with the larger listed serving size (and with seemingly higher amounts of less healthful nutrients such as sugar and sodium) actually had lower amounts of these nutrients in comparison to the cereal with the smaller listed serving size.

Conclusion: Attention to differences in the FUF and serving sizes was lacking. Participant inattention to the inequalities in serving sizes between cereals could mean they perceive them as being equal. Significant nutritional differences between products may be obscured by the failure of consumers to take different serving sizes into consideration.

Funding: None.

\section{P12 Do We Even Have Cancer: Cultural Beliefs About Health and Cancer Among Asian Indians}

Susmita Sadana, PhD, The Ohio State University; Peter Madril, $M S, L D, R D N$, The Ohio State University; Christopher Taylor, PhD, RD, LD, FAND, taylor.1043@osu. edu, The Ohio State University, 1675 Demaret Ln, Columbus, OH, 43228

Background: Asian Indians have been underrepresented in studies investigating knowledge and understanding of health and cancer, despite it being the second leading cause of mortality among this population. There is a paucity of data regarding the cultural perceptions of health and cancer in this population.

Objective: To gain an understanding of the cultural perceptions of health and cancer among Asian-Indian adults in an urban setting.

Study Design, Setting, Participants: Qualitative semi-structured interviews were conducted with AsianIndian adults $(\mathrm{n}=20), 25$ years and older. Participants had no prior diagnosis of cancer.

Measurable Outcome/Analysis: Individual interviews were conducted by a trained interviewer on Zoom ${ }^{\circledR}$. Verbatim transcripts were cross-checked for consistency prior to analysis. Transcript analysis was performed by 2 independent coders after training for qualitative analysis. Content analysis was used to identify themes using a grounded theory approach.
Results: Participants believed good health was associated with ability to perform daily activities, regular exercise and eating well. Lifestyle behaviors were considered important to health status. Healthy eating was stressed, which included fresh foods, traditional Indian foods, and a preference for vegetarian foods. Awareness of prevalence and implications related to cancer as it impacts the AsianIndian community. There was a general lack of knowledge around the aspects for cancer prevention, with a request for more targeted information.

Conclusion and Implications: Findings from this study underscore a gap in awareness of cancer risk among the Asian Indian community. More research is needed at a nation-wide level to corroborate these findings. Because of the general lack of knowledge and understanding of cancer, disseminating information about cancer risk and prevention among the Asian Indian community is important to address this disparity in health and disease.

Funding: School of Health and Rehabilitation Sciences, Office of Research's Discretionary Funding at the Ohio State University.

\section{P13 Evidence and Influences Regarding Food Insecurity Among Refugee in the USA: A Systematic Review}

Habiba Nur, MS, Habiba.nur@aggiemail.usu.edu, Department of Nutrition, Dietetics and Food Sciences, Utah State University, Logan, Utah, 8700 Old Main Hill, Logan, UT, 84322-8700; Abiodun Atoloye, PhD, Rudd Center for Food Policy and Obesity, University of Connecticut; Heidi Wengreen, $P h D, R D N$, Department of Nutrition, Dietetics and Food Sciences, Utah State University; Martha Archuleta, PhD, RDN, Department of Nutrition, Dietetics and Food Sciences, Utah State University; Mateja Savoie Roskos, PhD, MPH, RDN, Department of Nutrition, Dietetics and Food Sciences, Utah State University; Ron Munger, PhD, Department of Nutrition, Dietetics and Food Sciences, Utah State University; Gary Straquadine, PhD, College of Agriculture and Applied Sciences, Utah State University Eastern

Background: As refugees resettle in the USA, they face many challenges related to obtaining and preparing appropriate food. These challenges include having low incomes, lack of transportation, limited access to food assistance programs, and unfamiliar food environments, all of which increase the risk of food insecurity.

Objective: The objective of this review was to gain an understanding of the prevalence and impact of food insecurity among refugees in the USA.

Study Design, Settings, Participants: A systematic review of the literature was conducted in 10 electronic databases, including articles from 1980-2020. The following search terms were used: (food insecurity and refugee in the USA) or (food security and refugee in the USA). The quality of the selected articles was scored using the Hawker method (range: $9-36$ ). 